\title{
L'élève acteur des relations famille-école: stratégies de transmission des messages
}

\section{Walther Tessaro}

Familles et école sont aujourd'hui obligées de coopérer, ce qui conduit notamment à de nouvelles formes de relations entre parents et enseignants. Ces modifications nécessitent de reconsidérer la place de l'élève dans l'interface famille-école, en envisageant celui-ci à la fois comme produit et acteur des processus sociaux.

Notre recherche tente de mieux définir les stratégies mises en place par l'élève lors de la transmission de messages entre sa famille et l'école. L'efficacité de ces stratégies est directement liée à la connaissance scolaire, c'est-à-dire à la représentation des normes, des interdits, des rituels et des enjeux relationnels. Les résultats montrent l'importance du contenu du message à propos du type de stratégie mis en place: les élèves manifestent notamment un comportement plus audacieux lorsqu'ils se trouvent face à des messages qui contiennent des aspects évaluatifs.

Tout enseignant possédant une certaine expérience pédagogique pressent que de nombreuses interactions ont lieu dans la classe sans qu'il en ait connaissance. De même s'est-il rendu compte qu'il ne peut maîtriser entièrement les informations transmises aux parents par l'intermédiaire de l'élève. Si le contenu du message qui parvient à la maison est parfois différent de celui original, c'est parce que l'élève n'est pas un medium inerte (Perrenoud, 1987) et qu'il tente de contrôler la communication à son avantage.

Ce sont ces pratiques clandestines, qui ne relèvent pas d'une intention éducative, que nous avons cherché à mieux connaître dans cette recherche ${ }^{1}$. Nous désirons de la sorte apporter un éclairage sur des comportements encore mal connus et participer à une meilleure compréhension des expériences des élèves. Envisager ceux-ci comme des acteurs sociaux, c'est reconnaître l'existence d'un espace de liberté dans lequel ils peuvent agir. C'est aussi chercher à observer l'efficacité des comportements en se penchant sur les stratégies qu'ils mettent en place. 


\section{L'interface famille/école aujourd'hui}

Les standards élevés d'éducation ont pour conséquence, aujourd'hui, que familles et école sont obligées de coopérer (Cusin, 2000). Les prétentions nouvelles des parents constituent une pression externe qui nécessite de la part des enseignants une meilleure communication concernant notamment la gestion de la classe, les méthodes et les contenus d'enseignement. Cette nécessité s'inscrit dans une perspective plus globale de «rendre compte» (accountability) qui caractérise notre société actuelle (Montandon, 1987). Mais les modifications de cet interface famille/école ne vont pas sans redéfinir aussi la place de l'élève. Celui-ci pouvait en effet tirer profit d'une certaine séparation entre l'institution scolaire et l'institution familiale, en utilisant à son profit la marge de manœuvre existante. Un rapprochement ne peut donc que réduire sa liberté, ou du moins le forcer à repenser ses stratégies pour protéger ses intérêts contre les exigences des adultes.

Car c'est l'existence d'un interstice entre l'école et la famille qui permet progressivement à l'enfant de construire un univers qui lui soit propre. L'espace laissé par les parents et les enseignants, dont la complémentarité des rôles est essentielle aux yeux de l'enfant (Rayou, 1999), lui permet de grandir et de construire son autonomie. Dans ce processus, des attitudes systématiquement concordantes entre les deux parties, "l'effet de tenaille» dont parle Meirieu (1992), ou, à l'opposé, des désaccords menant à des situations conflictuelles peuvent avoir des conséquences néfastes. Si la tendance actuelle à une collaboration plus étroite entre enseignants et parents part d'une volonté avouée de venir en aide aux élèves, elle doit donc impérativement prendre en compte la place de ces derniers pour être vraiment efficace.

\section{L'enfant est acteur}

C'est dans un mouvement sociologique vers un retour à l'acteur (Sirota, 1993) et une nouvelle perspective de la socialisation que s'inscrit notre travail. Dans les contextes qui nous intéressent, c'est-à-dire l'école et la famille, l'enfant n'y est pas perçu comme un objet passif pris en charge par ces deux institutions mais comme un individu qui se constitue en sujet capable de s'approprier les événements qui le concernent et de leur donner du sens. Dans cette perspective interactionniste, l'enfant recrée continuellement le système social dans lequel il est amené à vivre et ceci dans les relations qu'il a avec ses pairs, ses parents et les enseignants. Il détermine donc autant la situation scolaire que la détermine le maître et autant la situation familiale que la déterminent ses parents.

La sociologie du métier d'élève (Perrenoud, 1984) s'est intéressée à la façon dont ce dernier répond aux attentes des enseignants et au sens qu'il donne à ses actions. Celles-ci ne s'observent cependant pas seulement à l'école, mais se poursuivent à l'extérieur et notamment dans le cadre des relations famille-école. 
L'élève y opère comme messager entre ces deux instances de socialisation, dans un rôle de go-between (Perrenoud, 1987) dont la double appartenance lui permet de mettre à profit des stratégies qui visent à sauvegarder des plages de liberté. La figure du go-between met en évidence une certaine autonomie de l'enfant vis-àvis des adultes et rend observables les nombreuses opportunités de négociation auxquelles il est confronté et qui font partie de ses expériences (Dubet, 1994).

La perspective que nous avons retenue permet d'envisager l'enfant à la fois comme produit et acteur des processus sociaux (Sirota, 1998). Il s'agit de se demander ce qu'il construit à l'intersection des instances scolaire et familiale, et non de s'interroger sur ce que fabriquent ces instances (Montandon \& Osiek, 1997). Cette approche constructiviste de la socialisation (De Quiéroz, 1995; Duru-Bellat \& Henriot-Van Zanten, 1992; Sirota, 1993; Van Haecht, 1990) est une perspective qui supplée au modèle classique, où les agents sociaux visent à intégrer les individus dans la société sans prêter à ces derniers une forme d'action déterminante.

Comme l'a souligné Touraine (1984), il y a toujours place pour des manifestations d'autonomie chez l'individu, même dans des situations de domination. Pour Crozier et Friedberg (1977), l'acteur ne peut exister que dans un espace de liberté défini par le système, mais celui-ci n'existe que par l'acteur qui lui donne vie et qui seul peut le changer. L'homme n'est pas totalement conditionné: capable de calcul et de manipulation, il s'adapte en fonction des circonstances et des partenaires. De ce fait, les comportements sont toujours actifs, car même la passivité relève d'un choix.

L'enfant n'est ainsi pas démuni face à l'influence des adultes et à leurs exigences implicites ou explicites. Que cela soit dans sa famille, dans sa classe ou entre ces deux lieux, il existe une marge de manoeuvre à l'intérieur de laquelle il peut élaborer des stratégies plus ou moins rationnelles, conscientes et cohérentes afin de faire face aux actions socialisatrices des adultes. Comme le relève Woods (1990), il n’y a pas de déterminisme culturel total. Dans la classe, des élèves peuvent déployer une grande ingéniosité pour ménager des intérêts personnels en opposition avec les influences de l'environnement.

\section{Actions et stratégies}

Les deux contextes éducatifs dans lesquels s'inscrit la socialisation de l'enfant peuvent être analysés selon un certain nombre de dimensions par rapport auxquelles il est appelé à se situer. Parmi ces dimensions, trois peuvent être retenues (Montandon \& Osiek, 1997): une logique de transmission, une logique d'organisation et une logique d'orientation. Face à ces trois logiques institutionnelles, l'enfant construit, en tant qu'acteur de sa propre socialisation, des expériences (Dubet, 1991, 1994; Montandon, 1995; Montandon \& Osiek, 1997; Sirota, 1998), qui englobent les représentations, les émotions et les actions. Les stratégies 
que l'élève élabore face aux situations auxquelles il est confronté quasi quotidiennement orientent ces dernières, même si elles sont parfois peu réfléchies.

Si l'élève entretient un rapport stratégique aux parents et aux enseignants, c'est qu'il n'est pas un simple réceptacle des influences familiales et scolaires. Partie prenante d'un réseau de relations interpersonnelles, l'enfant élabore des pratiques à partir de ses représentations de l'école (Charlot, Bautier \& Rochex 1992; Ogbu, 1992). Lui qui va et vient entre ces deux contextes culturels intervient sélectivement et activement dans les échanges entre enseignants et parents, composant ainsi avec sa double appartenance.

L'interactionnisme symbolique associe le terme de stratégie à l'acteur en tant que personne, qui peut agir sur les règles ou jouer avec celles-ci. La stratégie y est alors comprise comme un calcul et possède suffisamment de souplesse pour s'adapter aux incertitudes des situations.

A l'école obligatoire, l'élève a cependant peu de prise sur le système, tant individuellement que collectivement. Dans les rapports qu'il établit avec les adultes, on peut envisager, comme le fait Perrenoud (1996), qu'il est condamné «à des stratégies essentiellement défensives qui consistent à jouer avec les règles, à les contourner, à y échapper ou à en négocier l'application de cas en cas» (p. 102). Si ces stratégies apparaissent comme clandestines, elles sont le plus souvent tolérées car elles contribuent au bon fonctionnement de l'institution. Pour Perrenoud et Montandon (1988), toutes les actions ne sont cependant pas stratégiques, car «dans la vie quotidienne, chacun suit des routines, fait des choix qui relèvent davantage de l'habitus que du calcul stratégique» (p. 29). L'efficacité des stratégies est directement liée à la maîtrise de la culture scolaire, c'est-à-dire à la connaissance des normes, des interdits, des rituels, des influences et des enjeux relationnels qui sont en jeu (Vasquez, 1992).

\section{Stratégies et transmission des messages}

Plusieurs recherches ont mis en évidence des stratégies que les enfants disent mettre en place face aux parents ou face aux enseignants (Montandon \& Osiek, 1997; Nizet \& Hiernaux, 1984; Perrenoud, 1987, 1996; Woods, 1978, 1990). Parmi les différentes façons de regrouper ces stratégies, nous retiendrons la distinction qui est faite entre un conformisme vis-à-vis des adultes ou de l'institution (Montandon \& Osiek, 1997) et une certaine opposition ou résistance (Montandon \& Osiek, 1997; Nizet \& Hiernaux, 1984), manifestée de façon plus ou moins ouverte ou cachée. Il s'avère cependant que ces attitudes ne sont pas stables chez tous les acteurs et qu'une certaine alternance caractérise une partie de ceux-ci.

Ces attitudes se manifestent de différentes manières. Un comportement passif peut en effet viser à un certain conformisme comme il peut se manifester en situation de résistance. De même, une volonté de communiquer avec l'adulte ne 
signifie pas que l'on s'oppose à une décision ou à une information, mais peut tout aussi bien être une attitude valorisée par les parents.

Dans le champ des relations famille-école, certains auteurs (Collaud-Bounous, 1996; Pasquier, 1994; Perrenoud, 1987) définissent les stratégies mises en place comme actives ou passives par rapport au message à transmettre. Le regard ne porte plus spécifiquement, dans ce cas, sur la dynamique interactionnelle entre les élèves et les adultes, mais sur la variation de l'attitude en fonction du type de document à transmettre et du contenu de celui-ci. Cette caractéristique nous semble très pertinente car elle constitue une manière complémentaire d'aborder le champ des stratégies.

A partir des travaux décrivant les stratégies mises en place par les enfants dans les milieux familial et scolaire, nous avons défini deux axes permettant d'interpréter les attitudes de l'élève dans son rôle de messager entre la famille et l'école. Notre cadre conceptuel distingue le comportement face au message du comportement d'interaction interindividuelle. Le premier axe différencie les élèves faisant preuve de conformité, c'est-à-dire ceux qui ne tentent pas d'influencer la compréhension du message en adoptant une certaine neutralité, de ceux qui font preuve d'audace, c'est-à-dire les élèves qui agissent de façon plus ou moins conséquente sur les messages à transmettre. Le second axe différencie les élèves en fonction de leur propension à communiquer avec leurs parents: certains peuvent marquer une certaine ouverture s'ils facilitent ou provoquent la discussion, un certain repli s'ils évitent de parler du message et refusent le dialogue.

A partir de ces deux axes se dessinent quatre types de stratégies (figure 1), auxquels les élèves peuvent se rattacher d'une façon plus ou moins prononcée. L'adhésion se caractérise par la transmission des messages selon les attentes des adultes, avec une certaine disponibilité pour la discussion. Par les stratégies de persuasion, l'élève cherche à influencer l'interprétation des messages: il participe à leur formulation et argumente ses choix. Dans les stratégies de type soumission, les messages sont aussi transmis selon les attentes des adultes, mais les élèves évitent tout échange avec les parents ou l'enseignant. Enfin, l'élève altérant les messages en les oubliant, en les perdant ou en reportant leur transmission utilisera des stratégies de type manipulation.

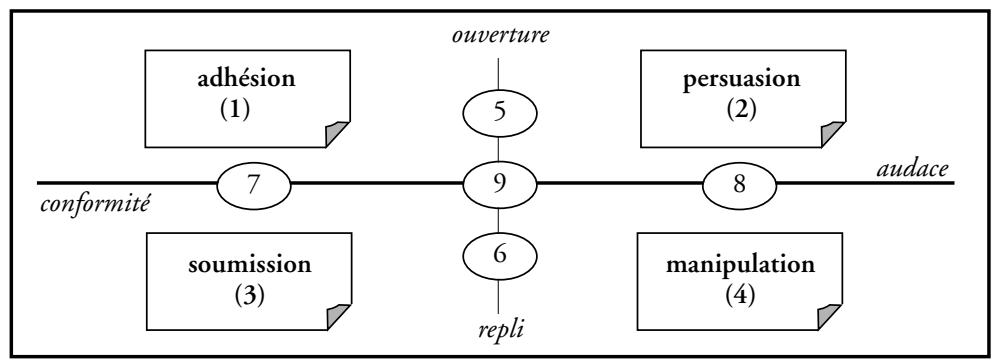

Figure 1: Types de comportements et modalités de stratégies

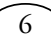


Les comportements des élèves ne se caractérisent cependant pas toujours par une orientation aussi claire vers l'un des deux pôles. Nous avons ainsi introduit, pour chacun des deux axes, une position neutre. Les trois positions par axe qui en découlent définissent, au total, neuf modalités de stratégies auxquelles un élève peut se rattacher: quatre types (adhésion, persuasion, soumission et manipulation) et cinq modalités appelées mixtes (numérotées de 5 à 9).

Tous les messages ne revêtant pas la même signification et ne suscitant pas le même intérêt, il nous est apparu nécessaire de les différencier selon des domaines scolaires communément acceptés. Ceux définis, au nombre de sept, regroupent la quasi totalité des messages qui circulent entre la classe et la famille: comportement, travail, devoirs, sorties et activités, rencontres, méthodes d'enseignement et administration.

Suite à cette première catégorisation selon le contenu des messages, nous avons considéré les domaines choisis selon une problématique transversale. La dimension qu'il nous importait d'étudier était celle de l'évaluation, dont l'importance dans le monde de l'école n'est plus à démontrer. Nous avons organisé les sept domaines en trois catégories. Ainsi, comportement et travail se regroupent en une même catégorie, car leur contenu comporte essentiellement des aspects évaluatifs. Devoirs et enseignement forment une deuxième catégorie, car ils peuvent comporter parfois des aspects évaluatifs, alors que rencontres, sorties et administration se distinguent par l'absence de ces aspects.

\section{L'évaluation comme moyen de communication}

Entre l'école et les familles, les moyens et les fréquences des contacts sont très diversifiés, comme l'ont montré Favre et Montandon (1989). Une part de ces contacts est directe, c'est-à-dire que les adultes entrent en contact directement. Ils peuvent être formels ou informels, individuels ou collectifs. S'ils sont les plus visibles et qu'ils ont été les plus étudiés, ils ne sont cependant pas les plus fréquents. En effet, la plupart des communications entre les familles et les enseignants se font de manière indirecte, par l'intermédiaire de l'enfant.

Parmi les moyens de communication, ce sont sans doute les évaluations qui revêtent le plus d'importance aux yeux des élèves. Transmettre une «note» ou toute autre forme d'appréciation à ses parents est l'occasion de pouvoir influencer la compréhension du message. Si l'évaluation peut être un moyen de communication sociale (Hadji, 1997) et s'inscrire dans un dialogue par les informations qu'elle fournit, l'enseignant n'a cependant que peu de prise sur la façon dont elle est rapportée aux parents. Le sens attribué au message, souvent implicite, peut varier plus ou moins fortement entre la classe et le domicile (Tessaro, 2000).

L'évaluation peut être interprétée comme une négociation sur un objet particulier et dans un environnement social donné (Hadji, 1997; Weiss, 1991). Les 
élèves constatent, dans leur cursus scolaire, que les enseignants ont leur propres habitudes, certains notant plus "généreusement» que d'autres en fonction de leur personnalité, de leurs convictions et du contexte (Barlow, 1977; Hadji, 1989). Elèves et parents sont particulièrement attentifs aux informations qui font part de la progression des apprentissages ou du niveau de maîtrise. Après quelques années de scolarité, les élèves ont appris à "calculer leurs chances, à mesurer les risques, et quelquefois même à tricher» (Weiss, 1992, p. 24). Certains deviennent de véritables experts dans leur rapport à la note, en calculant régulièrement leur moyenne, en dosant leur effort en fonction de l'évolution de celle-ci, en négociant ou en contestant un résultat ou une correction, en tombant malade le jour d'un contrôle difficile. Pour cela, ils savent qu'il faut connaître au mieux les habitudes et les critères de l'enseignant afin d'adopter les stratégies adéquates.

Comme le fait remarquer Perrenoud (1998), bien que l'évaluation transmise aux parents soit un système de communication souvent pauvre en informations, elle reste le lien le plus constant entre l'école et la famille. Les notes scolaires peuvent être parfois, les enseignants le savent bien, un moyen de discipline pour les parents (Dottrens, 1971): synonymes de récompenses lorsqu'elles sont satisfaisantes, de sanctions lorsqu' elles sont insuffisantes.

\section{Méthode de recherche}

Nous avons effectué notre récolte de données auprès de 84 élèves de 6ème année primaire, répartis dans quatre écoles publiques du canton de Genève. Le choix de ce degré scolaire se justifie notamment par l'expérience déjà importante du rôle de messager et par la quantité conséquente des informations à transmettre. C'est en Gème année primaire que se pose en outre à Genève la question de l'orientation vers les structures du secondaire inférieur, ce qui peut aussi favoriser le choix des stratégies.

Une volonté de cette recherche étant de donner la parole aux acteurs, l'entretien individuel nous a semblé de ce fait le moyen le plus adéquat afin de récolter les informations qui nous intéressaient. Pour chacun des sept domaines définis, un item du guide d'entretien a pris la forme d'un scénario décrivant une situation déjà vécue par l'élève ou du moins fortement envisageable par lui. Nous avons alors demandé à l'interlocuteur de choisir, parmi les réactions proposées, celle qui correspondait le mieux à la stratégie qu'il mettrait en place s'il était confronté à cette situation. Les réactions possibles correspondent aux types de stratégies définis dans le cadre d'analyse. Nous nous sommes inspiré, pour cette technique, de la méthodologie mise en place par Kellerhals, Perrin, SteinauerCresson, Vonèche et Wirth (1982).

Dans le tableau 1 figurent à titre d'exemple une question à choix multiple du domaine sorties/activités et les quatre propositions de réponse. 
Tableau 1: Exemple de question à choix multiple. Domaine: sorties, activités

\begin{tabular}{|c|l|}
\hline $\begin{array}{l}\text { Tu dois donner à tes parents des feuilles qui concernent la classe de neige. Parmi les choses que tu ne peux } \\
\text { pas prendre, figure le walkman (baladeur). Toi, tu adores l'utiliser. Que fais-tu? }\end{array}$ \\
\hline (1) & Je demande si on peut quand même écouter des cassettes au chalet \\
(2) & Tant pis, je le laisse à la maison et je serai content de le retrouver au retou \\
(3) & Je le prends en cachette \\
(4) & Avec mes camarades, j'essaie de faire changer le prof d'avis \\
\hline
\end{tabular}

(1) Adhésion; (2) Soumission; (3) Manipulation; (4) Persuasion

Des questions ouvertes étaient par ailleurs proposées en complément à chaque scénario, afin de définir plus précisément les stratégies de l'élève. Voici un exemple d'une question ouverte du domaine évaluation du comportement:

Supposons que tu aies fait quelque chose de grave à l'école, par exemple tu aies désobéi. Ton enseignant te donne une enveloppe fermée à transmettre à tes parents. Comment vas-tu t'y prendre?

Nous avons tout d'abord dépouillé toutes les questions à choix multiple, dont les réponses indiquaient l'orientation vers un des quatre types de stratégies. Cette démarche a permis d'obtenir un profil des stratégies utilisées par les élèves dans les sept domaines. Dans un second temps, nous avons utilisé les questions ouvertes proposées en complément aux vignettes afin de définir plus précisément cette orientation.

\section{Résultats}

Plusieurs méthodes d'analyse ont été utilisées dans notre recherche: analyse de variance, analyse des correspondances et analyse de tableaux de contingences. Cet article présentera seulement deux analyses jugées particulièrement intéressantes.

D’une manière générale, les résultats indiquent que les stratégies de type adhésion et persuasion ont été choisies dans presque deux tiers des situations. Ces deux types sont caractérisés par une ouverture dans l'attitude vis-à-vis des adultes. Ils correspondent, sans doute, aux comportements les plus valorisés par les parents et les enseignants, dont les attentes se manifestent quotidiennement de façon implicite ou explicite. Les choix manifestés montrent par ailleurs que les stratégies de type manipulation, par lesquelles les élèves altèrent les messages, sont quantitativement moins importantes.

Le tableau 2 présente la répartition des réponses, en pourcentage, entre les différents types de stratégies, par catégorie selon la dimension évaluative des mes- 
sages. On remarque en premier lieu le nombre important de modalités mixtes (voir figure 1), particulièrement dans la catégorie «aspects évaluatifs». Pour cette dernière, c'est avant tout la modalité 8 qui a été choisie, dénotant ainsi un comportement d'audace face aux messages. Il apparaît par ailleurs que le type de stratégie persuasion, manifestant aussi une certaine audace dans la transmission des messages, est dominant dans les catégories comportant toujours ou parfois des aspects évaluatifs. En outre, les types adhésion et soumission sont moins représentés dans la première catégorie (aspects évaluatifs) que dans les autres catégories, alors que c'est l'inverse pour le type manipulation, choisi notamment plus souvent dans le domaine comportement. On notera que les appréciations de ce domaine sont plus faciles à altérer, à oublier ou à modifier que celles relatives au travail, le plus souvent transmises dans un document officiel. Le lieu de transcription des informations semble donc aussi déterminer en partie le type de stratégies mises en place.

Tableau 2: Répartition des réponses, en pourcentage, entre les différents types de stratégie, par catégorie selon la dimension évaluative.

\begin{tabular}{|l|ccc|}
\hline Types de stratégies & $\begin{array}{c}\text { Aspects } \\
\text { évaluatifs } \\
\text { comp. / trav. }\end{array}$ & $\begin{array}{c}\text { Aspects pouvant } \\
\text { être évaluatifs } \\
\text { dev. / ens. }\end{array}$ & $\begin{array}{c}\text { Aspects non } \\
\text { évaluatifs } \\
\text { renc. / sort / adm. }\end{array}$ \\
\hline Adhésion & 17.5 & 21.5 & 33.3 \\
Persuasion & 26.0 & 29.0 & 23.0 \\
Soumission & 3.0 & 9.5 & 10.0 \\
Manipulation & 10.0 & 6.0 & 4.3 \\
\hline Mixtes & 43.5 & 34 & 29.4 \\
\hline
\end{tabular}

Domaines: comp. = comportement $;$ trav. $=$ travail; dev. $=$ devoirs; ens. = enseignement ; renc. $=$ rencontres $;$ sort $=$ sorties, activités $; \mathrm{adm} .=$ administration

Nous avons ensuite observé dans quelle mesure les types de stratégies sont associés aux performances scolaires des élèves. L'indicateur choisi pour cette variable est la moyenne générale annuelle obtenue par les élèves en fin de 5ème primaire (max. 6). Les élèves ont été regroupés en trois catégories: insuffisante (note inférieure à 4), suffisante (note entre 4,1 et 4,9) et élevée (note supérieure ou égale à 5). Nous avons calculé pour chacune d'elles la fréquence moyenne d'utilisation des types de stratégies, tous domaines confondus. Les résultats du tableau 3 indiquent une augmentation de la fréquence des stratégies de type persuasion entre les catégories «insuffisante» et "suffisante». Quant au type soumission, s'il a été nettement moins choisi, on retiendra que les élèves rencontrant le plus de difficultés scolaires l'ont préféré deux fois plus souvent que ceux qui ont les 
moyennes les plus élevées. Les données mettent aussi en évidence que ce sont les élèves qui obtiennent les meilleurs résultats scolaires qui tendent le plus fréquemment à altérer les messages (stratégies de type manipulation). Enfin, il apparaît que les élèves en difficulté scolaire manifestent généralement un comportement plus conforme face aux messages (stratégies de type adhésion et soumission), alors que ceux en réussite sont plus audacieux (stratégies de type persuasion et manipulation). Ce constat est soutenu par une analyse détaillée des stratégies mixtes.

Tableau 3: Fréquence moyenne d'utilisation des types de stratégies par les élèves, selon la catégorie des moyennes scolaires

\begin{tabular}{|l|ccc|}
\hline \multirow{2}{*}{ Types de stratégies } & \multicolumn{3}{|c|}{$\begin{array}{c}\text { Moyenne scolaire } \\
\text { Suffisante }\end{array}$} \\
\hline Adhésion & Insuffisante & 2.1 & 2.0 \\
Persuasion & 2.2 & 2.6 & 2.5 \\
Soumission & 1.4 & 0.4 & 0.5 \\
Manipulation & 1.0 & 0.4 & 0.8 \\
\hline Mixtes & 0.5 & 1.5 & 1.2 \\
\hline
\end{tabular}

$$
\chi^{2}=13.89 ; \mathrm{p}<.0032
$$

En analysant plus spécifiquement les résultats des domaines dont les messages contiennent des aspects évaluatifs, on relève que dans le domaine comportement, les stratégies de type adhésion sont davantage utilisées chez les élèves en difficulté. Par ailleurs, ce sont à nouveau les meilleurs élèves qui altèrent plus facilement les messages évaluatifs (type manipulation) et qui adoptent globalement un comportement plus audacieux.

\section{Discussion}

En s'exprimant sur les stratégies auxquelles ils ont recours dans les situations de la vie scolaire, les élèves nous ont dévoilé leur façon d'organiser les interactions avec les adultes et confirmé l'importance générale du contexte: le type de message, le destinataire, le domaine concerné ou le moment déterminent en effet les stratégies utilisées et rares sont les élèves qui ont recours de manière systématique au même type de stratégies. L'hétérogénéité des pratiques, révélée par les entretiens que nous avons menés, participe à la richesse de la dynamique scolaire.

Le fait que de nombreux élèves manifestent un comportement particulièrement audacieux dans la transmission des messages évaluatifs est à considérer avec attention. Bien que ces messages soient utilisés comme moyens de communication sociale (Hadji, 1997), l'information est filtrée par les messagers, qui modifient de façon plus ou moins conséquente les projets de l'enseignant. Ce constat 
semble confirmer l'importance de développer dès le plus jeune âge des compétences métacognitives et d'associer les élèves à la réflexion sur les objectifs pédagogiques. Il souligne aussi plus globalement la nécessité de prendre en compte les élèves dans l'étude de la socialisation (Dubar, 1991), la réalité d'une classe se caractérisant fréquemment par des situations de négociation et de co-construction (Allal, 2002). En modifiant le sens originel de certains messages, les élèves s'affirment comme acteurs sociaux d'un monde scolaire qu'ils participent aussi à transformer (Crozier \& Friedberg, 1977). Ce constat semble aussi confirmer que si ces messages revêtent une certaine valeur pour les élèves, c'est bien parce que les adultes les considèrent aussi importants (Montandon, 1991; Mouvement populaire des familles, 1978). Le style éducatif des parents et le type de communication privilégié peuvent faciliter ou rendre conflictuelle la relation au message évaluatif (Barlow, 1977), de même qu'ils peuvent avoir des conséquences sur l'estime de soi des enfants (Bélair, 1999).

Autre constat: ce ne sont pas les élèves en difficultés scolaires qui semblent avoir le plus souvent recours à un comportement audacieux. Nous pouvons faire ici l'hypothèse que les bons élèves doivent faire face à des attentes familiales plus élevées et qu'ils craignent d'autant plus fortement de décevoir leurs parents, les élèves ayant déjà rencontré des difficultés dans leur cursus ne semblant pas subir ce même type de pression. Néanmoins, il apparaît que l'audace des bons élèves face à de mauvais résultats se manifeste souvent par une argumentation étayée. S'agissant là d'une capacité valorisée par l'institution scolaire, il n'est dès lors pas étonnant qu'elle apparaisse chez ceux qui se sont approprié ces attentes (Charlot, Bautier \& Rochex, 1992) et qui y répondent le mieux.

Dans le champ des relations familles-école, une collaboration efficace entre adultes ne peut donc faire l'impasse d'une réflexion sur la place à donner à l'enfant. Plutôt que de viser à limiter sa marge de manœuvre, il s'agit de lui laisser l'opportunité de participer aux échanges. D'où l'intérêt, pour l'enseignant, d'impliquer l'élève dans ses apprentissages et dans son évaluation (Allal, 1999). Cette démarche peut les aider à devenir progressivement moins dépendants de la guidance de l'enseignant et des parents et à acquérir ainsi une certaine autonomie. Malheureusement, les tentatives de ce type ne donnent pas toujours des résultats satisfaisants car les attitudes souhaitées par l'enseignant ne conviennent pas à tous les élèves. Certains élèves ont en effet déjà développé tout au long de leur scolarité un rapport stratégique à l'évaluation qui leur permet de s'en sortir sans trop s'investir (Perrenoud, 1996).

\section{Conclusion}

Dans cette recherche, nous avons choisi de donner la parole aux enfants, pratique encore trop rare, afin qu'ils puissent s'exprimer sur leurs expériences sociales. Admettre qu'il sont capables d'introspection sur leur façon d'agir avec au- 
trui, c'est permettre qu'ils s'expriment sur leur métier d'enfant (Lahire, 1995; Rochex, 1995; Sirota, 1998). C'est aussi les considérer à la fois comme produits et acteurs à part entière des processus sociaux et non uniquement comme des êtres en devenir. Les compétences sociales que les enfants acquièrent à l'école leur permettront de faire face à de nouvelles expériences, et ceci dans d'autres organisations. Si les attitudes que valorise l'institution scolaire conditionnent fortement les compétences et les stratégies que les acteurs mobiliseront ensuite dans d'autres lieux, il nous semble important de mieux connaître l'émergence de celles-ci et d'observer comment s'articulent besoins individuels et objectifs organisationnels.

Notre recherche a visé en premier lieu à poursuivre les réflexions et les travaux effectués jusqu'ici concernant les stratégies que les élèves utilisent dans la relation famille-école. Elle comporte, dans cette optique, une dimension exploratoire qui ne peut qu'accroître les informations déjà existantes. Il a fallu pour cela élaborer un nouveau cadre conceptuel qui soit en continuité avec les observations menées dans ce champ, puis le mettre à l'épreuve dans le contexte choisi. Cette tentative se différencie des recherches précédentes non seulement dans l'organisation des types de stratégies qu'elle propose mais avant tout par la distinction et l'agencement des comportements qui définissent cette organisation.

Les analyses effectuées à ce sujet ont montré la pertinence des deux axes comportementaux, qui ont souvent apporté des précisions complémentaires aux analyses menées sur les stratégies des élèves. Elles ont permis par ailleurs de mieux saisir la complexité et l'enjeu des interactions entre l'élève et les adultes, parents ou enseignants. Si nous pouvons supposer que certains comportements sont davantage définis par la dynamique familiale et par les valeurs véhiculées à l'école, comme le comportement de conformité face aux messages, d'autres nous sont apparus plus représentatifs de l'individualité de l'élève, comme le comportement d'audace.

Une meilleure connaissance des expériences sociales des enfants participe bien sûr à une meilleure compréhension de cet objet sociologique. Cependant, une démarche comme celle que nous avons mise en place ne laisse entrevoir qu'une partie de l'univers secret de ces acteurs: celle qu'ils ont bien voulu nous faire partager. Nous souhaitons néanmoins que les informations recueillies aideront les enseignants à relativiser le contexte restreint de la classe et à privilégier un regard élargi sur l'environnement de leurs élèves. Plus concrètement, nous espérons que les questions auxquelles nous avons essayé de répondre dans ce travail, issues en partie d'une pratique d'enseignant, soient aussi un écho des préoccupations de certains collègues.

\section{Notes}

1 Cet article présente quelques résultats d'une recherche de thèse de doctorat en Sciences de l'éducation, réalisée sous la direction du professeur Linda Allal et soutenue en juin 2002 à l'Université de Genève (Tessaro, 2002). 


\section{Références}

Allal, L. (1999). Impliquer l'apprenant dans le processus d'évaluation: promesses et pièges de l'autoévaluation. In C. Depover \& B. Noël (Ed.), L'évaluation des compétences et des processus cognitifs: modèles, pratiques et contextes (pp. 35-55). Bruxelles: De Boeck.

Allal, L. (2001). Situated cognition and learning: From conceptual frameworks to classroom investigations. Revue Suisse des sciences de l'éducation, 23, 407-422.

Barlow, M. (1977). Notes et résultats scolaires de nos enfants. Paris: Centurion.

Bélair, L. (1999). L'évaluation dans l'école. Nouvelles pratiques. Paris: ESF.

Charlot, B., Bautier, E. \& Rochex J.-Y. (1992). Ecole et savoir dans les banlieues ... et ailleurs. Paris: Armand Colin.

Collaud-Bounous, S. (1996). Analyse de quelques relations famille-école du point de vue de l'enfant. Mémoire de licence en sciences de l'éducation. Université de Genève.

Crozier, M. \& Friedberg, E. (1977). L'acteur et le système. Paris: Seuil.

Cusin, C. (2000). Au cœur de redéfinitions. Linterface écolelfamille en Suisse. Rapport de tendance CSRE, 4. Aarau: Albdruck.

De Quiéroz, J.-M. (1995). L'école et ses sociologies. Paris: Nathan.

Dottrens, R. (1971). La crise de l'éducation et ses remèdes. Neuchâtel: Delachaux et Niestlé.

Dubar, C. (1991). La socialisation. Construction des identités sociales et professionnelles. Paris: Armand Colin.

Dubet, F. (1991). Les lycéens. Paris: Seuil.

Dubet, F. (1994). Sociologie de l'expérience. Paris: Seuil.

Durut-Bellat, M. \& Henriot-van Zanten, A. (1992). Sociologie de l'école. Paris: A. Colin.

Favre, B. \& Montandon, C. (1989). Les parents dans l'école Genève: Service de la recherche sociologique.

Hadji, C. (1989). L'évaluation, règles du jeu. Des intentions aux outils. Paris: ESF.

Hadji, C. (1997). L'évaluation démystifiée. Paris: ESF.

Kellerhals, J., Perrin, J.-F., Steinauer-Cresson, G., Vonèche, L. \& Wirth, G. (1982). Mariages au quotidien. Inégalités sociales, tensions culturelles et organisation familiale. Lausanne: Favre.

Lahire, B. (1995). Tableaux de familles. Paris: Gallimard, Le Seuil.

Meirieu, P. (1992). Les devoirs à la maison. Paris: Syros alternatives.

Montandon, C. (1987). L'essor des relations famille-école: problèmes et perspectives. In C. Montandon \& P. Perrenoud (Ed.), Entre parents et enseignants: un dialogue impossible? (pp. 23-47). Berne: Peter Lang.

Montandon, C. (1991). L'école dans la vie des familles. Genève: Service de la recherche sociologique.

Montandon, C. (1995). La socialisation scolaire: de l'expérience des enfants à l'analyse sociologique. Revue européenne des sciences sociales, 102, 95-119.

Montandon, C. \& Osiek, F. (1997). La socialisation à l'école du point de vue des enfants. Revue française de pédagogie, 118, 43-51.

Mouvement populaire des familles (1978). L'école en question. Un millier de parents s'expriment. Lausanne: Imprimeries populaires.

Nizet, J. \& Hiernaux, J.-P. (1984). Violence et ennui. Malaise au quotidien dans les relations professeurs-élèves. Paris: Presses universitaires de France.

Ogbu, J. (1992). Les frontières culturelles et les enfants des minorités. Revue française de pédagogie, 101, 9-26.

Pasquier, I. (1994). L'enfant messager entre sa famille et l'école: analyse des stratégies de quelques élèves du primaire genevois. Mémoire de licence en Sciences de l'éducation, Université de Genève.

Perrenoud, P. (1984). La fabrication de l'excellence scolaire. Genève: Droz.

Perrenoud, P. (1987). Le go-between: entre sa famille et l'école, l'enfant messager et message. In C. Montandon \& P. Perrenoud (Ed.), Entre parents et enseignants: un dialogue impossible? (pp. 49-87). Berne: Peter Lang. 
Perrenoud, P. (1996). Métier d'élève et sens du travail scolaire. Paris: ESF.

Perrenoud, P. (1998). L'évaluation des élèves. De la fabrication de l'excellence à la régulation des apprentissages. Entre deux logiques. Bruxelles: De Boeck.

Perrenoud, P. \& Montandon, C. (1988). Qui maîtrise l'école? Politiques d'institutions et pratiques des acteurs. Lausanne: Réalités sociales.

Rayou, P. (1999). La grande école. Approche sociologique des compétences enfantines. Paris: Presses universitaires de France.

Rochex, J.-Y. (1995). Le sens de l'expérience scolaire. Paris: Presses universitaires de France.

Sirota, R. (1993). Le métier d'élève. Revue française de pédagogie, 104, 85-108.

Sirota, R. (1998). L'émergence d'une sociologie de l'enfance: évolution de l'objet, évolution du regard. Education et sociétés, 2, 9-33.

Tessaro, W. (2000). Lévaluation comme moyen de communication entre familles et école. Quelle place pour l'élève? Bulletin du Gapp, 80, 18-21.

Tessaro, W. (2002). Lélève acteur de la relation famille-école: stratégies développées et facteurs motivationnels. Thèse de doctorat en sciences de l'éducation, Université de Genève.

Touraine, A. (1984). Le retour de l'acteur. Paris: Fayard.

Van Haecht, A. (1990). L'enfance: terre inconnue du sociologue. Bulletin AISLF, 6, 87-97.

Vasquez, A. (1992). Etudes ethnographiques des enfants d'étrangers à l'école française. Revue française de pédagogie, 101, 44-58.

Weiss, J. (1991). L'évaluation: un problème de communication. Cousset: Del Val.

Weiss, J. (1992). L'enseignant «au cœur froid» ou l'objectivité en évaluation. Mesure et évaluation en éducation, 14, (4), 19-31.

Woods, P. (1978). Negociating the demands of schoolwork. Journal of curriculum studies, 10, (4), 309-327.

Woods, P. (1990). L'ethnographie de l'école. Paris: Colin.

Mots clés: comportement, élève, enseignant, famille, milieu scolaire, norme sociale, relation parents-école

\section{Der Schüler als Akteur in der Beziehung Schule-Familie: Mitteilungsübermittlung und entwickelte Strategien}

\section{Zusammenfassung}

Heutzutage sind Familien und Schulen verpflichtet zusammenzuarbeiten, was zu neuen Beziehungsformen zwischen Eltern und Lehrern führt. Diese Veränderungen erfordern eine Überdenkung der Stellung des Schülers in der Schnittstelle zwischen Familie und Schule, indem der Schüler gleichzeitig als Produkt und Akteur der sozialen Prozesse betrachtet wird.

Unsere Forschung versucht, die vom Schüler angewandten Strategien anlässlich der Mitteilungsübermittlung zwischen Schule und Familie genauer zu bestimmen. Die Wirksamkeit dieser Strategien ist unmittelbar mit den Schulkenntnissen verbunden, d.h. mit den Vorstellungen von Normen, Verboten, Ritualen und Beziehungsgeflechten.

Die Ergebnisse zeigen die Wichtigkeit des Mitteilungsinhalts bezüglich des angewandten Strategietypus: Die Schüler zeigen ein kühneres Verhalten, wenn sie mit Mitteilungen konfrontiert sind, die Einschätzungsaspekte enthalten. 
Schlagworte: Beziehung Schule-Familie, Familie, Lehrer, Schulmilieu, Schüler, soziale Norm, Verhalten

\section{L'allievo protagonista delle relazioni famiglia-scuola: strategie di trasmissione di messaggi}

\section{Riassunto}

Scuola e famiglia sono oggigiorno tenute a cooperare e ciò comporta nuove forme di relazione tra insegnanti e genitori. Questi mutamenti richiedono una riconsiderazione del ruolo dell'allievo al punto d'incontro tra scuola e famiglia: egli va visto tanto come attore quanto come risultante dei processi sociali. La nostra ricerca ha cercato di definire le strategie che l'allievo mette in atto nella trasmissione die messaggi tra la sua famiglia e la scuola. L'efficacia di tali strategie è direttamente legata alla conoscenza della scuola, in particolare alle rappresentazioni di norme, proibizioni, rituali e relazioni. I risultati mostrano il nesso tra contenuti del messaggio e scelta della strategia comunicativa: gli allievi si mostrano più audaci quando sono confrontati con messaggi di carattere valutativo.

Parole chiave: alunno, ambiente scholastico, comportamento, famiglia, insegnante, norma sociale, rapporti scuola-famiglia

\section{The pupil as part of the relation between family and school: transmission of messages and developed strategies}

\section{Abstract}

Today, families and schools are under obligation of cooperating, which notably induces new forms of relations between parents and teachers. These modifications make it necessary to reconsider the child's position in the family-school interface, envisaging him at the same time as product and actor of the social processes involved.

Our research attempts to better define the strategies developed by the pupil in the transmission of messages between his family and school. The efficiency of these strategies is directly linked to the knowledge of school ways, meaning representations of norms, forbidden aspects, rituals and relational stakes. Results indicate the importance of the message's content about the type of strategy developed: in particular, pupils manifest a more daring behaviour when they find themselves confronted to messages that contain evaluative aspects.

Key words: behaviour, family, pupil, school environment, school-familiy relationship, social norm, teacher 
